\title{
Concomitant Occurrence of Fenestrated Internal Jugular Vein and Sub-omohyoid External Jugular Vein: A Case Report
}

\author{
Sulochana Sakthivel ${ }^{1}$, Suman Verma ${ }^{1}$ \\ ${ }^{1}$ Associate Professor, Jawaharlal Institute of Postgraduate Medical Education and Research, Pondicherry, India
}

\section{Abstract}

External jugular vein is the superficial vein of the neck and is prone to variations. Multiple internal jugular veins are incidental findings that present as a duplication or fenestration. We encountered a unilateral fenestrated internal jugular vein and a bilateral variation in the course of external jugular vein, during a cadaveric dissection. The external jugular vein, after its formation, crossed the sternocleidomastoid muscle and pierced the investing cervical fascia of the posterior triangle. It traversed deep to the inferior belly of omohyoid muscle to enter the subclavian triangle and terminated by draining into the subclavian vein on the left side, and at the angle between the internal jugular vein and the subclavian vein on the right side. The fenestrated internal jugular vein on the left side divided into a small medial and large lateral division which reunited at the level of the tendon of omohyoid muscle and drained into the subclavian vein. Only the medial division of the internal jugular vein received tributaries in the neck. Awareness of the multiple variations of the jugular veins would be valuable during surgical approaches to the neck. Present report aims to be useful for vascular surgeons, radiologists, and intensivists as well.

Keywords: Jugular veins, neck, omohyoid, fenestration.

Corresponding Author: Dr. Suman Verma, Associate Professor, Department of Anatomy, JIPMER Academic center, Jawaharlal Institute of Postgraduate Medical Education and Research, Pondicherry, India - 605006

Email: suman2v@gmail.com

Received: August 2018

Accepted: September 2018

\section{Introduction}

External jugular vein (EJV) is the superficial vein of the neck formed by the union of the posterior division of the retromandibular vein with the posterior auricular vein, near the angle of mandible. It runs downwards in neck, crosses the sternocleidomastoid muscle to reach the fascia over posterior triangle. At the root of the neck, it pierces the deep fascia to drain into the subclavian vein. ${ }^{[1]}$ Being easily accessible, it is favored for cannulation, and also used as a graft in carotid endarterectomy. ${ }^{[2]} \mathrm{EJV}$ is known to be prone to variations. A variation in its formation can be accompanied by a course deep to omohyoid muscle in the neck. $^{[2]}$

Internal jugular vein (IJV) drains the skull, brain, neck and superficial parts of the face. It is the continuation of the sigmoid sinus and unites with the subclavian vein to form the brachiocephalic vein. ${ }^{[1]}$ Multiple IJVs are rare, incidental finding. Mostly, two patterns have been described in literature. One is duplication of IJV, in which both the medial and lateral divisions of IJV drain separately in to the subclavian vein while the other is fenestration of IJV, where both the divisions reunite and drain as a single IJV. ${ }^{[3]}$
Another classification describes multiple IJVs as duplication and takes into account the level of duplication of IJV in relation to digastric muscle and hyoid bone. ${ }^{[4]}$ Incidence of fenestrated IJV varies from $0.4 \%$ to $1 \%$ and is linked with phlebectasia or added vascular anomalies. ${ }^{[5,6]}$

\section{Case Report}

During routine dissection in a 50-year-old male cadaver for undergraduate teaching program, variation in the external and internal jugular veins was encountered. On both the sides of neck, union of the posterior division of retromandibular and posterior auricular veins formed the EJVs. The left EJV, after its formation at the apex of parotid gland, crossed the middle third of sternocleidomastoid muscle and pierced the deep fascia in the occipital triangle. Few small tributaries from submental region formed the anterior jugular vein, rest continued as a large submental vein to open into the EJV. The part of the EJV before it perforated the deep fascia was tortuous and received a large submental vein. Further, the left EJV traversed deep to the inferior belly of omohyoid muscle and entered the subclavian triangle. After a downward and slight medial course, it opened into left subclavian vein [Figure 1]. Right 
EJV also took a similar sub-omohyoid course to open at the angle between the right IJV and subclavian vein.

On the left side, the IJV divided into a small medial and large lateral division at the level of the tendon of digastric muscle [Figure 2]. From above downwards, medial division of IJV received the anterior division of retromandibular vein, facial vein, lingual vein and the superior thyroid veins. Lateral division of IJV which was larger in caliber did not receive any tributaries in the neck. Both the divisions of IJV reunited at the level of the tendon of omohyoid muscle and drained into the subclavian vein. The middle thyroid vein drained just distal to the union of medial and lateral divisions of IJV. There was no variation in the formation or termination of the right IJV.

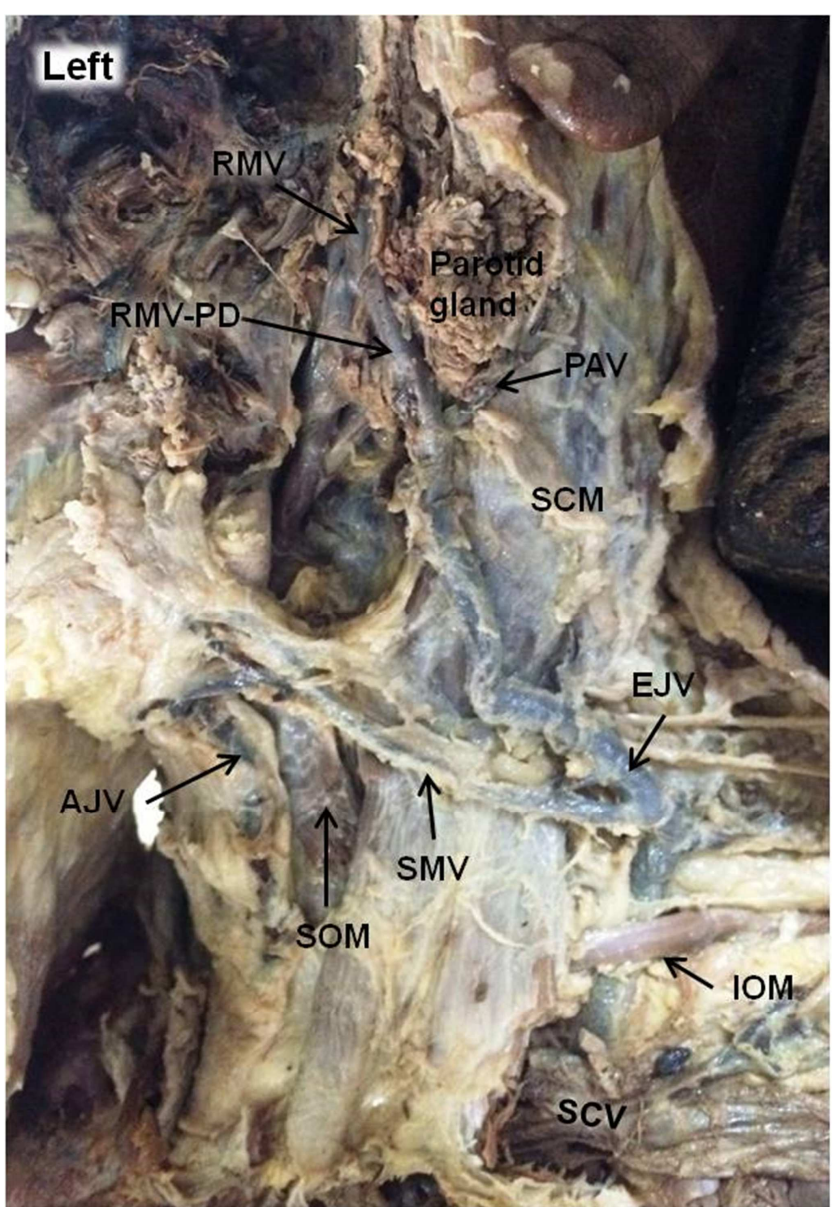

Figure 1: Sub-omohyoid left external jugular vein (mandible has been removed). RMV-retromandibular vein, PD-posterior division, PAV-posterior auricular vein, SCMSternocleidomastoid, EJV-external jugular vein, SMVsubmental vein, SOM-superior belly of omohyoid, IOMinferior belly of omohyoid, AJV-anterior jugular vein, SCVsubclavian vein.

\section{Discussion}

Multiple IJV is a rare and usually a chance finding. Downie et al. classified the multiple IJVs into two different vascular patterns, fenestration and duplication. ${ }^{[3]}$ The present case was identified as fenestration pattern, since two divisions of the IJVs joined and drained as a single vein into the subclavian vein. Nayak et al. proposed three morphological patterns of duplicated IJV. ${ }^{[4]}$ In type A, duplication of IJV starts above the inferior border of posterior belly of digastric and united cranial to or at the level of central tendon of omohyoid muscle. The accessory nerve usually passes between the two IJVs. In type B, the duplication extends inferior to omohyoid tendon whereas in type $\mathrm{C}$, duplication occurs around the level of hyoid bone and the lateral component of IJV might traverse outside the carotid sheath, in the posterior triangle and enter the carotid sheath at the root of the neck. ${ }^{[4]}$ In accordance with the classification by Nayak et al., the present case falls under type A pattern.

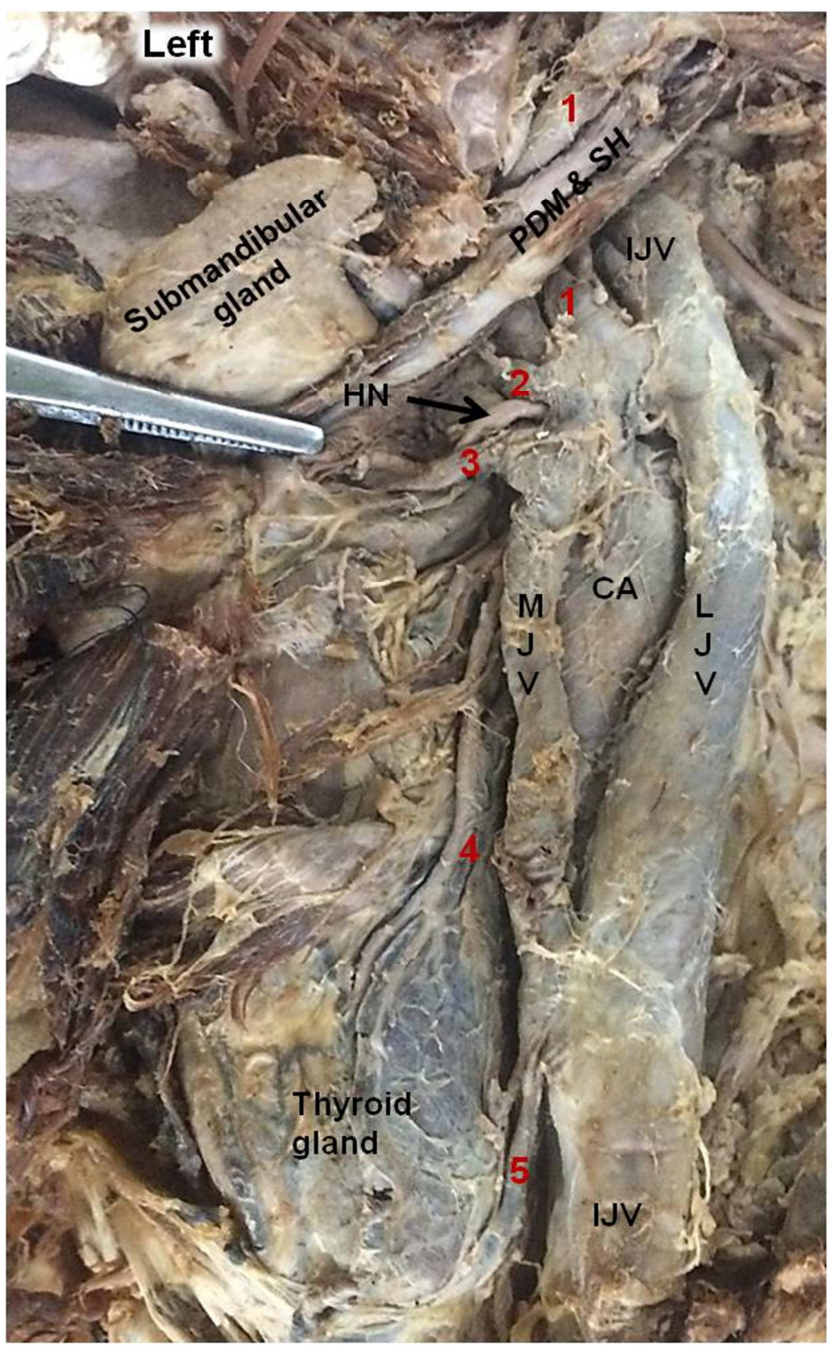

Figure 2: Fenestrated left internal jugular vein(IJV). MJVmedial jugular vein, LJV-lateral jugular vein, CA-carotid artery, HN-hypoglossal nerve, PDM-posterior digastric muscle, SH-stylohyoid, 1-anterior division of retromandibular vein, 2facial vein, 3-lingual vein, 4-superior thyroid vein, 5-middle thyroid vein.

Prades et al. observed the spinal accessory nerve to pass anterior to the medial IJV and posterior to the lateral IJV, in all the three cases encountered by them. ${ }^{[5]}$ A study by Saman et al., demonstrated that the spinal accessory nerve crossed the IJV anteriorly in $80 \%$, posteriorly in $19 \%$ of the specimens and in one case, it traversed between the 
bifurcated IJV. ${ }^{[7]}$ Dogan et al. reported that the ansa cervicalis and the inferior belly of omohyoid traversed between the two divisions of the IJV. ${ }^{[8]}$ In the present report, ansa cervicalis and the omohyoid were anterior to the fenestrated IJV. Besides, the spinal accessory nerve was posterior to both the medial and lateral IJVs, which is similar to that of a case reported by Alaani et al. ${ }^{[9]}$

The sub-omohyoid course of EJV is a rare anatomic arrangement and seldom reported in literature. On the basis of recent literature search, only one case of sub-omohyoid EJV has been reported so far and none with concurrent fenestrated IJV. The sub-muscular course would put EJV under increased stress and would make it liable for compression against the underlying brachial plexus. ${ }^{[2]}$ Such pressure on the vein would also explain the tortuosity of proximal EJV we observed in this case.

Variations in the vascular pattern of the neck might be explained by the intussusception branching remodeling. The intraluminal pillars that are formed in the arteries and veins lead to remodeling of the vessels by expansion or pruning of the branches. Intussusceptive angiogenesis is influenced by changes in the blood flow and expression of vascular endothelial cell growth factors (VEGF). Over-expression of VEGF causes enlargement of vessels whereas downregulation results in pruning of vessels. Vascular maturation becomes dormant if the VEGF remains constant. ${ }^{[10]}$

Variant anatomy of the neck veins depends on the outcome of anastomoses between the veins that develop into IJV and EJV. IJV develops from the precardinal veins that drains the head and neck, and is continuous with the primary head vein cranially. The ventral pharyngeal vein (VPV) initially drains into common cardinal vein, but with the growth of the neck, it terminates into the precardinal vein that becomes the IJV later. The VPV forms the linguofacial vein which anastomoses with the primitive maxillary vein, situated superolateral to VPV, to become the facial vein. Facial vein receives the retromandibular vein from the temporal region and drains into the IJV. ${ }^{[1]}$ EJV develops as a tributary of the cephalic vein and anastomoses with the anterior facial vein. The cephalic vein forms a venous ring around the clavicle and is connected to the precardinal vein. The deep part of the ring develops into subclavian vein and receives the EJV. The anterior connection between the anterior facial vein and EJV disappears later. ${ }^{[1]}$

\section{Conclusion}

The present case of sub-omohyoid EJV and fenestrated IJV is a rare anatomical presentation. The sub-muscular course, apart from placing the EJV under increased pressure, is also likely to cause difficulties in cannulation and in exposure of the vein during surgical procedures. Fenestrated IJV might result in significant clinical consequences during central venous access, radical neck dissections, or in diagnosing neck pathology.

\section{References}

1. Standring S. Gray's Anatomy: The Anatomical Basis of Clinical Practice. 41th ed. London. Elsevier Churchill Livingstone; 2016. p 459, 619-620.

2. Sirasanagandla SR, Narendra P, Satheesha NB, Somayaji SN, Kumar MR Bhat, Surekha DS, Jyothsna P. Unusual formation and Sub-omohyoid course of external jugular vein. J Surg Academia. 2013;3(2):89-91.

3. Downie SA, Schalop L, Mazurek, JN, Savitch G, Lelonek GJ Olson TR. Bilateral Duplicated Internal Jugular Veins: Case Study and Literature Review. Clin. Anat. 2007;20(3):260-266.

4. Nayak SP, Ashraf M, Dam A, Biswas J. Internal jugular vein duplication: Review and classification. Indian J Surg Oncol. 2017;8(2):222-226.

5. Prades JM, Timoshenko A, Dumollard JM, Durand M, Merzougui $\mathrm{N}$, Martin C. High duplication of the internal jugular vein: clinical incidence in the adult and surgical consequences, a report of three clinical cases. Surg Radiol Anat. 2002;24:129-132.

6. Contrera KJ, Aygun N, Ward BK, Gooi Z, Richmon JD. Internal jugular vein duplication and fenestration: Case series and literature review. The Laryngoscope. 2016;126:1585-1586.

7. Saman M, Etebari P, Pakdaman MN. Anatomic relationship between the spinal accessory nerve and the jugular vein: a cadaveric study. Surg Radiol Anat. 2011;33:175-179

8. Dogan UN, Cicekcibasi FE, Fazliogullari Z, Yilmaz MT, Uysal II, Salbacak A. Unilateral variations of vessels and nerves in the neck. Int. J. Morphol. 2010;28(3):963-966.

9. Alaani A, Webster K, Pracy JP. Duplication of internal jugular vein and relation to the spinal accessory nevre. J Oral Maxillofac Surg. 2005;43:528-531

10. Djonov VG, Baum O, Burri PH. Vascular remodeling by intussusceptive angiogenesis. Cell Tissue Res. 2003;314:107-117.

Copyright: () the author(s), publisher. Academia Anatomica International is an Official Publication of "Society for Health Care \& Research Development". It is an open-access article distributed under the terms of the Creative Commons Attribution Non-Commercial License, which permits unrestricted non-commercial use, distribution, and reproduction in any medium, provided the original work is properly cited.

How to cite this article: Sakthivel S, Verma S. Concomitant Occurrence of Fenestrated Internal Jugular Vein and Sub-omohyoid External Jugular Vein: A Case Report. Acad. Anat. Int. 2018;4(2):7-9.

DOI: dx.doi.org/10.21276/aanat.2018.4.2.3

Source of Support: Nil, Conflict of Interest: None declared. 